\title{
Reassortant human group C rotaviruses in Hungary
}

Szilvia Marton ${ }^{a}$, Judith Deák ${ }^{\text {b }}$, Renáta Dóró ${ }^{a}$, Tünde Csata a , Szilvia L. Farkas ${ }^{\text {a }}$, Vito Martella ${ }^{\mathrm{c}}$, Krisztián Bányai ${ }^{\mathrm{a}}$

${ }^{a}$ Institute for Veterinary Medical Research, Centre for Agricultural Research, Hungarian Academy of Sciences, Budapest, Hungary

${ }^{b}$ Institute of Clinical Microbiology. University of Szeged, Szeged, Hungary

'Department of Veterinary Medicine, University of Bari Aldo Moro Valenzano, Italy

Corresponding author

Word count in manuscript

Keywords
Krisztián Bányai, Institute for Veterinary Medical Research, Centre for Agricultural Research, Hungarian Academy of Sciences, H-1143 Budapest, Hungária krt. 21, Hungary; Tel.: +36 1467 4060, Fax: +36 1467 4076; Email: bkrota@hotmail.com

1007

Rotavirus $C$; whole genome sequencing; semiconductor sequencing; phylogenetic analysis; molecular epidemiology 


\section{Dear Sir,}

Group C rotaviruses (species Rotavirus $C, \mathrm{RVC}$ ) are characterized with a non-enveloped, triple-layered capsid that encases an 11 -segmented double-stranded RNA genome. RVCs are classified in the genus Rotavirus, family Reoviridae (Estes and Greenberg, 2013). Epidemiological investigations worldwide have revealed that the prevalence of RVCs in gastroenteritis is lower than that of group A rotaviruses (RVA). Difference in detection rates of RVC among studies is pending on choice of method, study year and area, epidemiological settings and age group. Apart from the sporadic cases reported from many countries worldwide large outbreaks associated with RVC have been also reported, predominantly from Japan (Adah et al., 2002; Baek et al., 2013; Bányai et al., 2006; Esona et al., 2008; Hamano et al., 1999; Kumazaki and Usuku, 2014; Medici et al., 2009; Rodger et al., 1982; Schnagl et al., 2004). Methods to detect and differentiate RVC infection from gastroenteritis caused by other rotavirus species rely on antigen detection, genomic RNA fingerprinting and reverse transcription (RT)-PCR coupled with nucleotide sequencing (Fujii et al., 1992; Guovea et al., 1991; Khamrin et al., 2011; Meleg et al., 2008; Rodger et al., 1982).

RVC infection has been reported in a few cases in Hungary using genomic RNA fingerprinting and RT-PCR (Bányai et al., 2006; Szucs et al., 1987). Sequencing and phylogenetic analysis of the VP7, VP4, VP6 and NSP4 genes uncovered great genetic relatedness of the Hungarian RVC strains to other common human RVCs (Bányai et al., 2006). The number of human RVC strains with whole genome sequence is scanty. Available genome sequence information suggests that at least two major human lineages can be distinguished based on backbone gene combinations. One cluster includes strains detected in various areas of the globe including the historic strain, Bristol, whereas the other cluster includes fewer strains detected during the past decade in Far East Asian countries (Yamamoto 
et al., 2011). Although the zoonotic potential of RVCs is not fully understood, partial genome sequence information suggests that it may occur from swine to human, which may further increase the strain diversity in humans (Gabbay et al., 2008).

Recently, we have set up a whole genome sequencing based strain surveillance of human RVAs. When processing data obtained for the sample set collected during 2012-2013 we serendipitously identified RVC sequences in stool samples selected originally based on positive antigen test results for RVA (Table). In the 2012-2013 RVA surveillance study, a total of 392 human stool samples were processed for unbiased detection of RVA genotypes using the protocol described earlier (Dóró et al., 2014). In brief, laboratory methods included RNA extraction from stool suspensions followed by random primed RT-PCR. Two-hundred base pair DNA libraries were prepared for clonal amplification and semiconductor sequencing on Ion 316 chips. Next-generation sequencing data were processed by trimming, de novo assembly and reference mapping onto a large collection of viral sequences retrieved from GenBank using the modules in the CLC Genomics Workbench (http://www.clcbio.com).

Following de novo and metagenomic assemblies of sequence reads a total of four samples were found to contain RVC genomic RNA. All four samples were detected during 2013 in the south-eastern region of Hungary (Table). Of these, one sample had low number of RVC specific sequence reads $(n=14)$ and was not used for additional analyses. Another three samples yielded sufficient sequence reads to be able to assemble the entire coding regions and even the (near) full genome sequence. The genomes of strains, RVC/Humanwt/HUN/ERN6210/2013/G4P[2], RVC/Human-wt/HUN/ERN6216/2013/G4P[2] and RVC/Human-wt/HUN/ERN6233/2013/G4P[2] were assembled from 13474, 9085, and 13262 sequence reads, respectively (GenBank accession numbers KP776576-KP776608).

To determine the genetic relationship among the Hungarian RVCs and those detected in other geographical areas reference sequences were retrieved from GenBank 
(http://www.ncbi.nlm.nih.gov/genbank). Phylogenetic analysis was carried out with the maximum-likelihood and neighbor-joining algorithms as implemented in MEGA6 (http://www.megasoftware.net). Best fit substitution models (NSP1, GTR+G+I; NSP2, T92+I; NSP3, TN93+I; NSP4, T92+G; NSP5, HKY+G; VP1, TN93+G+I; VP2, GTR+G+I; VP3, $\mathrm{GTR}+\mathrm{G}+\mathrm{I} ; \mathrm{VP} 4, \mathrm{GTR}+\mathrm{G} ; \mathrm{VP} 6, \mathrm{~T} 92+\mathrm{G}+\mathrm{I} ; \mathrm{VP} 7, \mathrm{~T} 92+\mathrm{G}$ ) were selected based on the BIC (Bayesian Information Criterion) values. The robustness of trees was tested by 1000 bootstrap replications

The analyses of the $11 \mathrm{RVC}$ genes showed very close genetic relationship among the three Hungarian RVC strains ( $>99 \%$ nt similarities with 0 to 5 point mutations along each segment). Additionally, all three strains shared $\geq 99 \% \mathrm{nt}$ sequence identity with RVC strains collected during the 2000s in Japan and China with the exception of the NSP4 gene. These Far East Asian strains were reported to have several distinguishing features from the globally major variant of human RVCs, including the putative unique genotype of the VP3 gene as well as minor sequence variations in other genes (Yamamoto et al., 2011). All three Hungarian RVCs clustered with the Far East Asian strains in most gene phylogenies. The only exception was seen in the NSP4 gene phylogeny showing a closer relationship to the globally spread variants of human RVCs (Fig).

In Hungary, RVC infections are thought to be rare (Bányai et al., 2006; Szucs et al., 1987). RVCs in this study were serendipitously identified in stool samples positive for RVA. The detection rate of RVCs among samples selected for RVA strain monitoring from the south-eastern region of Hungary was $0 \%$ ( 0 of 94) in 2012 and $7.1 \%$ (4 of 56) in 2013. This finding was somewhat unexpected and could be associated with a local outbreak or small epidemic during the winter-spring season of 2013. Unfortunately, as we included in the analysis only RVA-positive samples, it was not possible to determine the true prevalence of RVCs in the study period and its relevance to disease. 
To our knowledge this is the first study from Europe to report the occurrence of reassortant human RVCs bearing the genetic backbone of the Asian lineage of human RVCs. The introduction of this newly described human RVC lineage in Hungary was accompanied with reassortment involving the NSP4 gene. The question, whether in Europe this lineage is currently co-circulating with the globally common lineage or novel reassortant strains may have replaced the historic RVC lineage over time, is unclear. The apparent changes in the epidemiology of human RVCs is a novel finding that warrants the extension of RVC surveillance using whole genome sequence data in future molecular epidemiological investigations.

\section{Acknowledgements}

The study was supported by the Hungarian Scientific Research Fund (OTKA, T100727) and the Momentum program awarded by the Hungarian Academy of Sciences.

\section{References}

Adah, M.I., Wade, A., Oseto, M., Kuzuya, M., Taniguchi, K., 2002. Detection of human group $\mathrm{C}$ rotaviruses in Nigeria and sequence analysis of their genes encoding VP4, VP6, and VP7 proteins. J. Med. Virol. 66, 269-275.

Baek, I.H., Than, V.T., Kim, H., Lim, I., Kim, W., 2013. Full genomic characterization of a group C rotavirus isolated from a child in South Korea. J. Med. Virol. 85, 1478-1484.

Bányai, K., Jiang, B., Bogdán, A., Horváth, B., Jakab, F., Meleg, E., Martella, V., Magyari, L., Melegh, B., Szucs, G., 2006. Prevalence and molecular characterization of human group C rotaviruses in Hungary. J. Clin. Virol. 37, 317-322. 
Dóró, R., Mihalov-Kovács, E., Marton, S., László, B., Deák, J., Jakab, F., Juhász, Á., Kisfali, P., Martella, V., Melegh, B., Molnár, P., Sántha, I., Schneider, F., Bányai, K., 2014. Largescale whole genome sequencing identifies country-wide spread of an emerging G9P[8] rotavirus strain in Hungary, 2012. Infect. Genet. Evol. 28, 495-512.

Esona, M.D., Humphrey, C.D., Dennehy, P.H., Jiang, B., 2008. Prevalence of group C rotavirus among children in Rhode Island, United States. J. Clin. Virol. 42, 221-224.

Estes, M.K., Greenberg, H.B., 2013. Rotaviruses. in: D.M. Knipe, P.M. Howley (Eds.), Fields Virology, 6th edition. Lippincott - Williams and Wilkins Publishers, Philadelphia PA. pp. $1347-1401$

Fujii, R., Kuzuya, M., Hamano, M., Yamada, M., Yamazaki, S., 1992. Detection of human group C rotaviruses by an enzyme-linked immunosorbent assay using monoclonal antibodies. J. Clin. Microbiol. 30, 1307-1311.

Gabbay, Y.B., Borges, A.A., Oliveira, D.S., Linhares, A.C., Mascarenhas, J.D., Barardi, C.R., Simões, C.M., Wang, Y., Glass, R.I., Jiang, B., 2008. Evidence for zoonotic transmission of group C rotaviruses among children in Belém, Brazil. J. Med. Virol. 80, 1666-1674.

Gouvea, V., Allen, J.R., Glass, R.I., Fang, Z.Y., Bremont, M., Cohen, J., McCrae, M.A., Saif, L.J., Sinarachatanant, P., Cau,1 E.O., 1991. Detection of group B and C rotaviruses by polymerase chain reaction. J. Clin. Microbiol. 29, 519-523.

Hamano, M., Kuzuya, M., Fujii, R., Ogura, H., Mori, T., Nakayama, T., Yuen, E., Katayama, K., Mitsunobu, Y., Inoue, K., 1999. Outbreak of acute gastroenteritis caused by human group C rotavirus in a primary school. Jpn. J. Infect. Dis. 52, 170-171. 
Khamrin, P., Okame, M., Thongprachum, A., Nantachit, N., Nishimura, S., Okitsu, S., Maneekarn, N., Ushijima, H., 2011. A single-tube multiplex PCR for rapid detection in feces of 10 viruses causing diarrhea. J. Virol. Methods. 173, 390-393.

Kumazaki, M., Usuku, S., 2014. Epidemiological and genetic analysis of human group C rotaviruses isolated from outbreaks of acute gastroenteritis in Yokohama, Japan, between 2006 and 2012. Arch. Virol. 159, 761-771.

Matthijnssens, J., Ciarlet, M., McDonald, S.M., Attoui, H., Bányai, K., Brister, J.R., Buesa, J., Esona, M.D., Estes, M.K., Gentsch, J.R., Iturriza-Gómara, M., Johne, R., Kirkwood, C.D., Martella, V., Mertens, P.P., Nakagomi, O., Parreño, V., Rahman, M., Ruggeri, F.M., Saif, L.J., Santos, N., Steyer, A., Taniguchi, K., Patton, J.T., Desselberger, U., Van Ranst, M.,. 2011. Uniformity of rotavirus strain nomenclature proposed by the Rotavirus Classification Working Group (RCWG). Arch Virol. 156, 1397-1413.

Medici, M.C., Abelli, L.A., Martinelli, M., Martella, V., Dettori, G., Chezzi, C., 2009. Molecular characterization of group $\mathrm{C}$ rotaviruses detected in children in Italy. J. Clin. Virol. $44,62-65$.

Meleg, E., Bányai, K., Martella, V., Jiang, B., Kocsis, B., Kisfali, P., Melegh, B., Szucs, G., 2008. Detection and quantification of group C rotaviruses in communal sewage. Appl. Environ. Microbiol. 74, 3394-3399.

Rodger, S.M., . Bishop, R.F, Holmes, I.H., 1982. Detection of a rotavirus-like agent associated with diarrhea in an infant. J. Clin. Microbiol. 16, 724-726.

Schnagl, R.D., Boniface, Cardwell, K., P., McCarthy, D., Ondracek, C., Coulson, B., Erlich, J., Morey, F., 2004. Incidence of group C human rotavirus in central Australia and sequence variation of the VP7 and VP4 genes. J. Clin. Microbiol. 42, 2127-2133. 
Szucs, G., Kende, M., Uj, M., 1987. Atypical human rotaviruses in Hungary. Ann. Inst. Pasteur/Virol. 138, 391-395.

Yamamoto, D., Ghosh, S., Kuzuya, M., Wang, Y.H., Zhou, X., Chawla-Sarkar, M., Paul,

S.K., Ishino, M., Kobayashi, N., 2011. Whole-genome characterization of human group C rotaviruses: identification of two lineages in the VP3 gene. J. Gen. Virol. 92, 361-369. 


\section{Figure legends}

Fig. Phylogenetic trees obtained by the neighbor-joining method for all 11 genes using representative international strains and the Hungarian human RVCs. A bovine RVC strain was used as outgroup throughout the analyses. Hungarian strains are highlighted. Bootstrap values $>60$ are shown in branch nodes. Scale bars are proportional to the genetic distance; numbers above the scale bars indicate the number of substitutions per sites. Putative main lineages (solid lines) and/or sub-lineages (dashed lines) of human RVCs are indicated on the right margin of each tree. In the VP3 gene phylogeny, solid lines on the margin separate putative genotypes. Strain nomenclature recommended by Matthijnssens et al. (2011) is implemented using the $\mathrm{G}$ and $\mathrm{P}$ genotype designations as recommended by Yamamoto et al. (2011). (Phylogenetic trees obtained by the maximum-likelihood method showed similar tree topologies; data not shown) 
CIHUman-wt'GBRiBristo|/1988/G4P[2] RVICiHuman-wtiJPN/OH567/2003/G4P[2]

RVCHUman

RVClHuman-wtJ.JPN:BK0830/2008iG4P[2]

RVCIHuman-wUCHNVINR001/2007/G4P[2]

RVCIHUman-WtHHUNIERNE233/2013/GAPR

RVCIHuman-wt'HUNIERN6210/2013/G4P[2]

7 RVC:Human-wtHUNIERN621E/2013/G4P[2] Outgroup
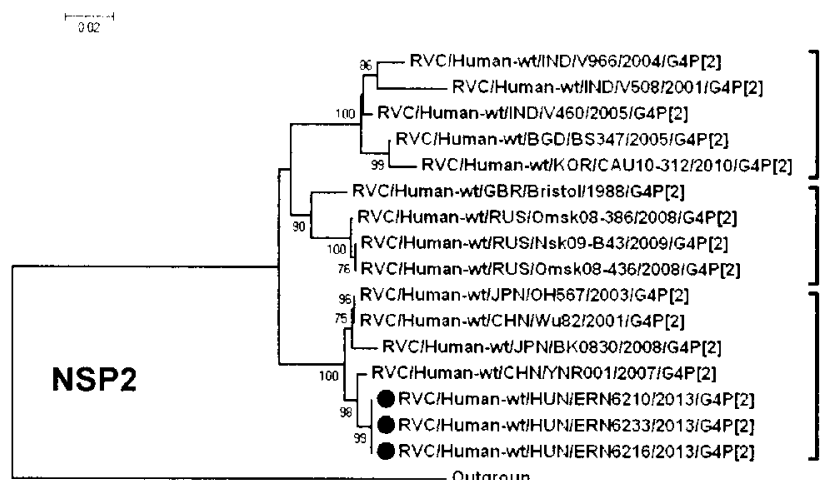
Outgroup

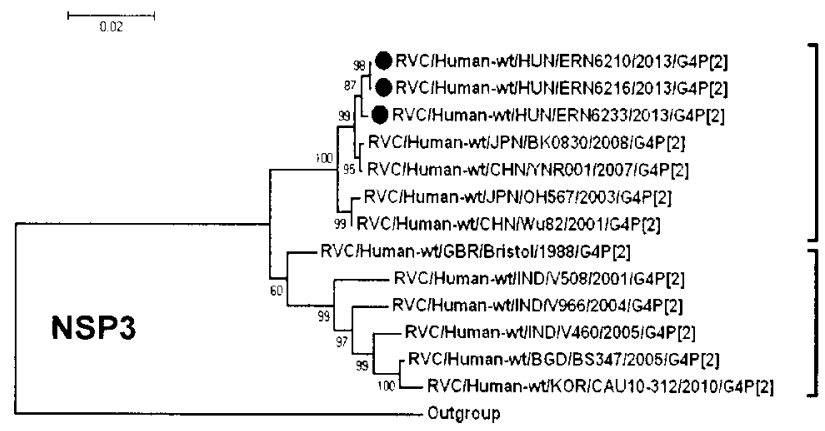

RVC/Human-wtRUSiOmsk08-386/2008/G4P[2] RVC/Human-wtiRUSiOmsk08-436/2008/G4P[2] RVCiHuman-wt/RUSINsk08-3414:2008/GXP[X] ORVGIHUman-WtiHUNIERN6233/2013/G4P[2] 61 7 RVC/Human-wtHUNERN621012013/G4P [2] 6.7 RVCIHuman-Wt'HUNJERN6216/2013/G4P[2] L RVCIHuman-wtIRUSINsk09-843/2009/64P[2]

RVCIHuman-wtilNDIN508/2001/G4P[2]

95 L RVCIHuman-wt/NDEN966/2004/G4P[2] RVCIHUman-wtINON46012005/G4P[2] 77 RVC/Human-wt/BGDIB S347/2005/G4P[2]

99 LVCJHuman-WtKKOR/CAU10-312/2010/G4P[2]

- RVClHuman-wtiCHN/208/1994/G4P[2]

_RVCIHuman-wt/GBR/Bristoll1988/G4P[2] RVC/Human-wtiXXX/Ehime9301/XXXXJGXP[X]

- RVCIHuman-wtJJPNN'06-1/2006iG4P[2]

RVC/Human-wtiJPNIOH567/2003/G4P[2]

RVCIHuman-wtiCHN/WU82/2001/G4P[2]

RVC/Human-WtTURIGUP 188/2005/G4P[2]

RVCiHuman-WUtJPNN/BK0830/2008/G4P[2]

RVCIHuman-wt/JPN/N09-1/2009/GAP[2]

RVCiHuman-wtJJPNIV12-12012/G4P[2]

RVCiHuman-wtJJPN/12-2/2012/G4P[2]

RVCHUMman-Wt/CHNYYNR0012/2007/GAP[2]

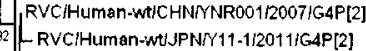

NSP4

[RVCIHuman-wtiJPN/Y08-212003/G4P[2]

2 - RVCIHuman-wtiBRAlIAL-RC32/2008/G4P[2]

-RVCIHuman-wtiJPNN/08-4/2008/G4P[2]

RVCIHuman-wtIJPNrro8-1/2008/G4P[2]

RVC/Human-wiJJPNN08-3/2008/G4P[2] Outgroup

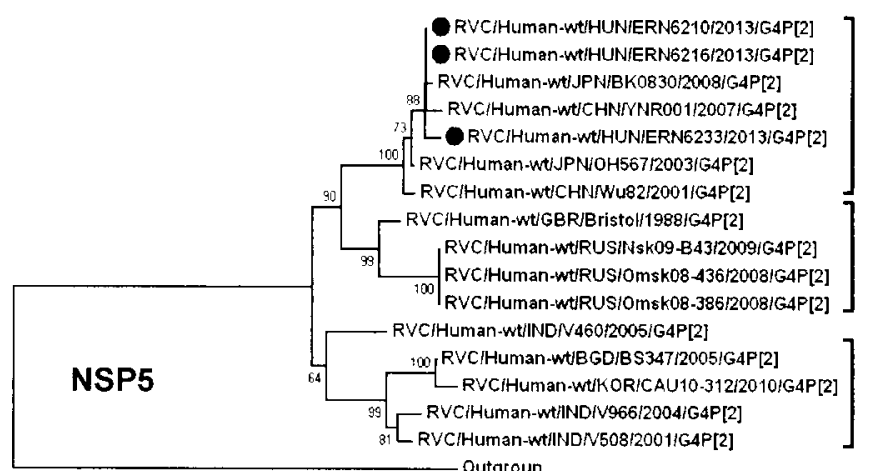
Outgroup 
RVCHUman-wtiCHNWW 82/2001/G4P[2]

[RVCIHuman-wtJPNIOH567/2003/G4P[2]

100 RVCIHuman-wt/CHNNNR001/2007/G4P[2]

- RVCIHuman-wtiJPNIBK0830/2008/G4P[2]

98 RRVCIHuman-wtiHUNIERN6233/2013GG4P[2]

RVCIHuman-wt/HUNIERN6216/2013/G4P[2]

${ }_{90}$ RVCiHuman-wt'HUNIERN621012013/G4P[2]

- RVCIHuman-wtGBR/Bristol/1988/G4P[2]

- RVCIHuman-wtilNDN508/2001/G4P[2]

VP1

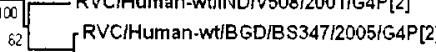

${ }_{100}$ RVCIHuman-wt/KORICAU10-31212010iG4P[2]

Outgroup

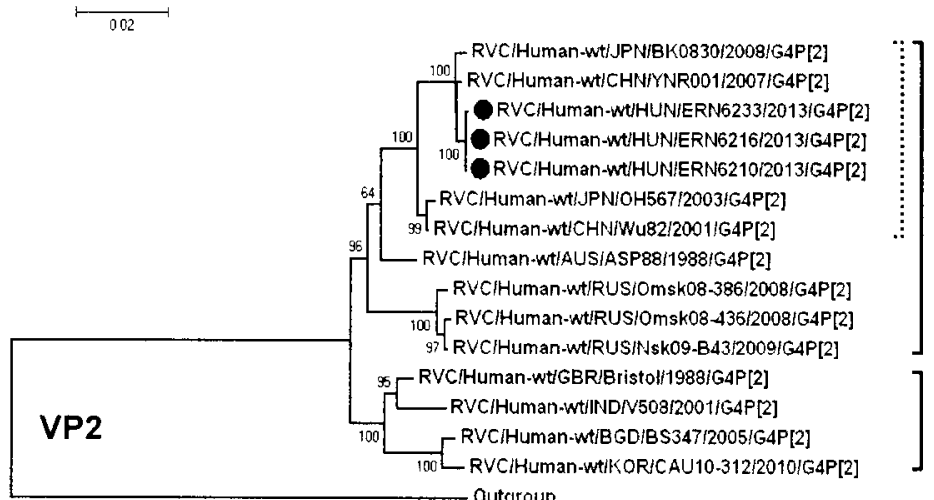

Outgroup

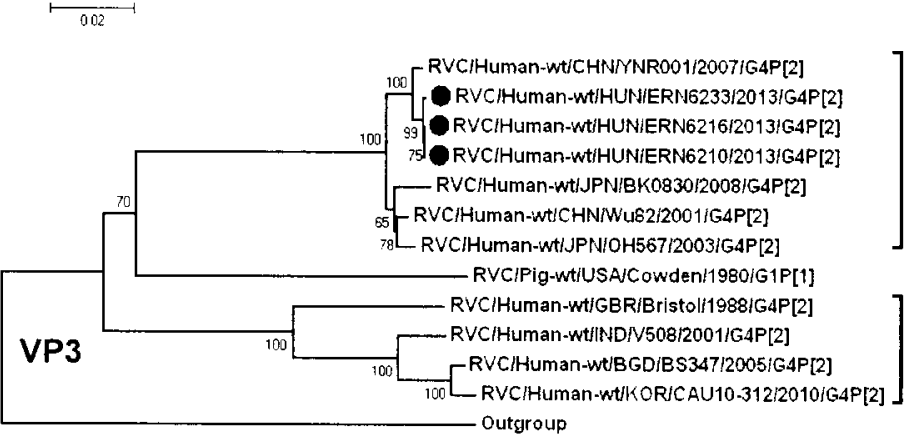

RVCIHuman-wtJJPNYY12-12012/G4P[2] RVC/Human-WtJJPN/12-2/2012/G4P[2] RVCIHUman-WUJPNIYO8-4/2008/G4P[2] 100 RVC/Human-wtJJPN/V08-3/2008/G4P[2] RVC/Human-wtiJPN/Y08-1/2008/G4P[2]

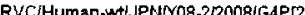
RVC/Human-WUCHNTRO0012007/G4P[2] -RVCIHUman-WtiJPN/Y11-112011/G4P[2] [ RVCFHuman-wt/HUNIERNG233/2013/G4P[2]

RVC/Human wtHHUNIERN6216/2013/64P[2]

Q RVCHUMTan-wtHHUNEREN621012013!G4P[2]

RVC!HUMan-NTIESPIBCNOII997/G4P[2]

RVC/Human-wtIINDN508/2001/G4P[2]

-RVC/Human-wtiNNDIV966/2004/G4P[2]

RVCitiuman-wtillNDN46012005iG4P[2]

RVCIHUman-wtiBGDIBS347/2005/G4P(2)

[ RVCIHuman-WtIKORICAU10-312/2010/GAP[2]

RVCIHuman-wtiNGAIModuganari/1999/G4P[2]

100 RVCIHuman-wt/NGAiJajeril/1999/GAP[2]

10: RVCIHuman-wtIESPIBCN6/1999-2000/G4P[2]

${ }_{100}^{100}$ RVCIHUman-wUESPIBCNN21/1990-2000/G4P[2]

og [RVIHuman-wtiGBR/Bristol/1988/G4P[2]

_ RVCIHuman :wtiBRA/Bele m[1989/GAP[2]

L RVC/Human-wt/AUSICHRVIA87J/1987/G4PI

VP4

WAOUCHRVABTJ

- RVCIHuman-wtJJPNr/06-12006/G4P[2]

RVCiHuman-wt/CHN/208/1994/G4P[2]

100 RVC/Human-wt/JPNIEK0830/2008/G4P[2]

RVCIHUman-wt/JPN/V09-112009/GAP[2]

RVC/Human-WtTURIGUP188/2005/G4P[2]

RVCIHuman-wtiCHNIWUP212001/G4P[2]

${ }_{90}$ RVCIHuman-wt:JPNIOH567/2003/GAP[2] Outgroup

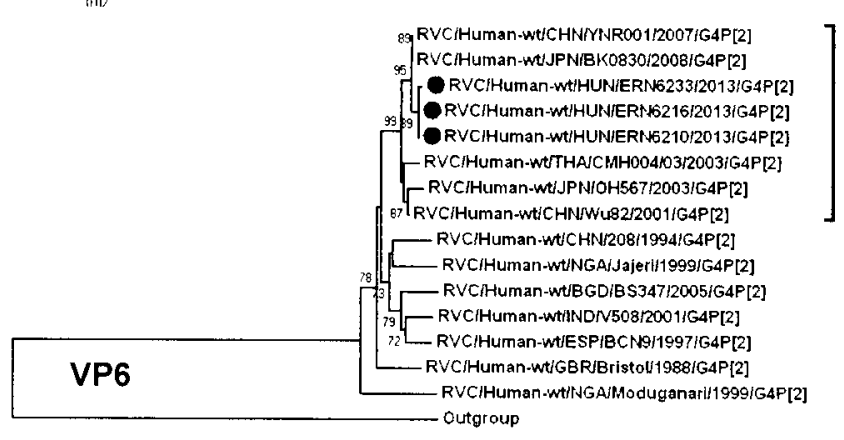

RVCIHUman-wUICHNMTNR0012007/G4P[2] RVCRHuman-wtJPN/BKO830/2008/G4P [2] RVCIHuman-wt/HUNIERN6233/2013/G4P[2]

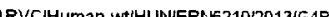

WVIJuman-wt/CHN/WLA2/2001/G4P Outgroup 


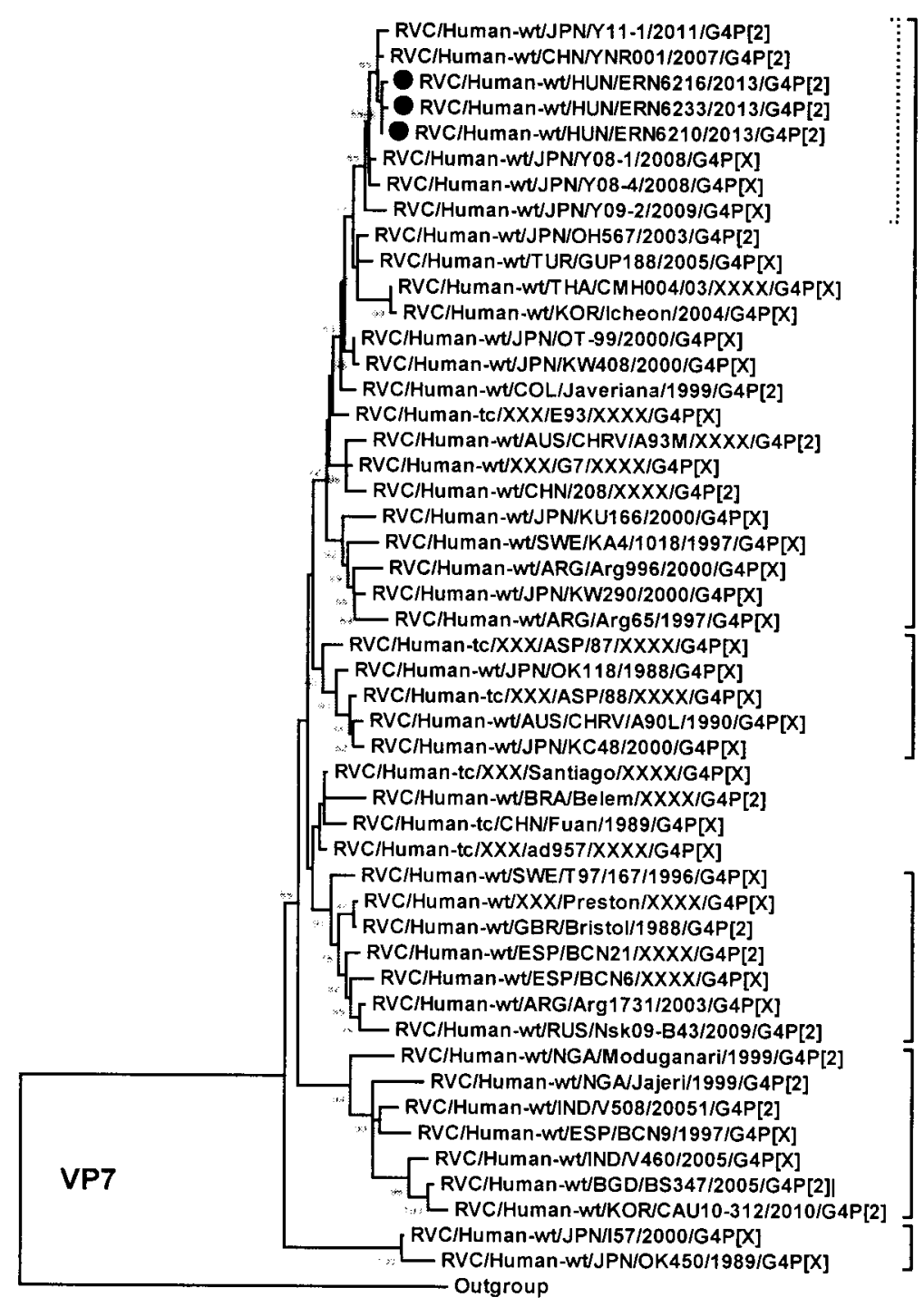


Table Background information of diarrheic patients whose stool specimens were positive for human RVC by viral metagenomics.

\begin{tabular}{lllllll}
\hline Patient & Sample & Setting & Age & Gender & Symptoms & Living area \\
& collection & & & & & \\
\hline 1 & Jan 2013 & Outpatient & $4 \mathrm{y}$ & $\mathrm{M}$ & $\mathrm{D}+\mathrm{V}$ & Urban \\
2 & Jan 2013 & Inpatient & $74 \mathrm{y}$ & $\mathrm{M}$ & $\mathrm{D}$ & Rural \\
3 & Jan 2013 & Inpatient & $9 \mathrm{y}$ & $\mathrm{F}$ & $\mathrm{D}+\mathrm{V}$ & Urban \\
4 & Apr 2013 & Inpatient & $5 \mathrm{y}$ & $\mathrm{F}$ & $\mathrm{D}$ & Rural
\end{tabular}

$\mathrm{D}$, diarrhea; $\mathrm{D}+\mathrm{V}$, diarrhea and vomiting 


\section{Accepted Manuscript}

Reassortant human group $\mathrm{C}$ rotaviruses in Hungary

Szilvia Marton, Judith Deák, Renáta Dóró, Tünde Csata, Szilvia L. Farkas, Vito Martella, Krisztián Bányai

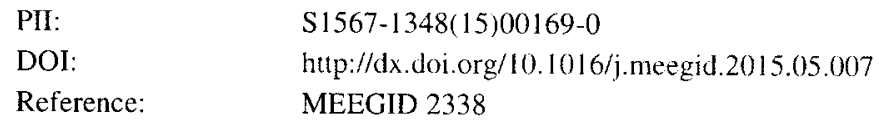

Please cite this article as: Marton, S., Deák, J., Dóró, R., Csata, T., Farkas, S.L., Martella, V., Bányai, K., Reassortant human group $\mathrm{C}$ rotaviruses in Hungary, Infection, Genetics and Evolution (2015), doi: http://dx.doi.org/10.1016/ j.meegid 2015.05.007

This is a PDF file of an unedited manuscript that has been accepted for publication. As a service to our customers we are providing this early version of the manuscript. The manuscript will undergo copyediting, typesetting, and review of the resulting proof before it is published in its final form. Please note that during the production process errors may be discovered which could affect the content, and all legal disclaimers that apply to the journal pertain. 